\title{
Composición de los desembarques de la pesca artesanal en los puertos de Boca Parita y El Agallito, Pacífico panameño
}

\section{Composition of artisanal fishing landings in the ports of Boca Parita and Agallito, Panamanian Pacific}

\author{
Yarkelia Aneth Vergara-Frías ${ }^{1}$, Yolani Aibeth Robles-Pineda ${ }^{2 *}$ y Ángel Javier Vega ${ }^{2,3}$
}

\begin{abstract}
RESUMEN
Para los puertos de Boca de Parita y El Agallito, en el Pacífico panameño, se contabilizó la biomasa desembarcada entre el 2011 y 2015, adicionalmente, se desarrollaron muestreos de desembarque para identificar, medir y pesar las especies y cuantificar su aporte en número y peso, además de registrar los precios pagados al pescador. Los resultados indican que entre ambos puertos se comercializaron 2760 toneladas de productos pesqueros, donde el 74.5\% correspondió a Boca de Parita y el $25.5 \%$ a El Agallito. En los desembarques se identificaron 38 especies, donde las más abundantes: Cynoscion phoxocephalus (Sciaenidae), Scomberomorus sierra (Scombridae) y Lutjanus guttatus (Lujanidae). Más de $90 \%$ del producto analizado fue de primera y segunda calidad, según precio de comercialización. La distribución de tallas, en las especies con un tamaño de muestra superior a 100 individuos, y para las cuales se encontró información sobre la media reproductiva $\left(\mathrm{L}_{50}\right)$, refleja que solo en Caranx vinctus y Centropomus medius, más del $80 \%$ de los desembarques estuvo compuesto por ejemplares maduros, en Lutjanus guttatus y Scomberomorus sierra el 49\% y 53\%, y en Cynoscion phoxocephalus, Caranx caballus, y Cynoscion albus, el porcentaje fue de $19,5 \%$ y $0 \%$, respectivamente. Esto indica que es necesario establecer políticas de manejo, donde se instauren regulaciones de tallas de captura y zonas de pesca que garanticen que la actividad esté dirigida a la captura de individuos que se hayan reproducido, como primer paso para garantizar la sostenibilidad de la pesca artesanal en el golfo de Parita.
\end{abstract}

Palabras claves: pesca artesanal, pesca comercial, biomasa desembarcada, Bahía de Parita, talla de captura

1 Autoridad de los Recursos Acuáticos de Panamá, ciudad de Panamá, Panamá. yvergara52@hotmail.com ORCID: http://orcid.org/0000-0003-2818-6743

2 Centro de Capacitación, Investigación y Monitoreo de la Biodiversidad en el Parque Nacional Coiba, Universidad de Panamá (CCIMBIO-CRUV-UP), Calle 10, Santiago de Veraguas, Panamá. angel.vega@up.ac.pa ORCID: http:// orcid.org/0000-0002-9535-3348,yolany.robles@up.ac.pa* ORCID: http://orcid.org/0000-0003-4140-9235

3 Estación Científica Coiba AIP. Parque Nacional Coiba. 


\section{ABSTRACT}

For the ports of Boca de Parita and El Agallito, in the Panamanian Pacific, biomass landed was counted for the period between 2011 and 2015. The individuals in these landings were also identified, measured and weighed, and the prices paid by intermediaries to fishermen were recorded. The results of these efforts indicate that a total of 2760 tons of fishery products from both ports were marketed, with $74.5 \%$ of the total landed at Boca Parita and the remainder at El Agallito. In the landings, 38 species were identified, of which the most abundant: Cynoscion phoxocephalus (Scianidae), Scomberomorus sierra (Scombridae) and Lutjanus guttatus (Lutjanidae). For both ports, over $90 \%$ of landings consisted or first and second quality products in terms of market price. Size distributions for species with a sample size of more than 100 individuals for which data on mean reproductive size (L50) was also available indicate that in the cases of Caranx vinctus and Centropomus medius, over $80 \%$ of landings consisted of mature individuals, while for Lutjanus guttatus and Scomberomorus sierra 49\% and 53\%, respectively, of individuals were mature. The percentages of mature individuals of Cynoscion phoxocephalus, Caranx caballus and Cynoscion albus were 19, 5 and $0 \%$, respectively. This indicates a need to establish management policies which regulate catch size and/or establish no-take zones that guarantee the capture of individuals that have already reproduced, as a first step in ensuring the sustainability of artisanal fishery in the Parita Gulf.

Keywords: Artisanal fishery, commercial fishery, landed biomass, Parita Bay, catch size

\section{INTRODUCCIÓN}

El manejo de la pesca artesanal en el Pacífico panameño presenta una serie de inconvenientes, tales como: bases de datos deficientes, estrategias pesqueras carentes de validez científica, conflictos en el uso de los recursos y su ineficiente (Maté, 2005). Parte de esta problemática se ha tratado de resolver a través del desarrollo de planes de manejo en áreas protegidas, en los cuales se incluyen planes de aprovechamiento pesqueros sostenibles (Autoridad Nacional del Ambiente, 2009).

De manera general, para el manejo pesquero, este tipo de debilidades crean incertidumbre sobre el estado de los recursos (Arreguín-Sánchez \& Arcos-Huitrón, 2011), e inciden sobre una gestión exitosa de la actividad, la cual depende del cumplimiento de múltiples objetivos, enmarcados en aspectos biológicos, ecológicos y socioeconómicos (Liu et al. 2016).

Es así como, estudios sobre pesquerías buscan llenar vacíos de información sobre los sistemas de pesca, que han llevado al desarrollo de investigaciones que aporten datos sobre las especies objetivo, los ecosistemas y las operaciones de las flotas (González-Becerril et al. 2000; Guzmán-Mora \& Molina-Ureña, 2008; Vázquez-Hurtado et al. 2010; Gutiérrez-Zavala \& Cabrera-Manci1la, 2012; Harper et al. 2014). 
Por otro lado, con la revisión de datos históricos de pesca, se ha logrado, en algunos casos, reconstruir el comportamiento de las capturas, todo esto con el propósito de mejorar la calidad de la información que pueda utilizar el administrador pesquero (Valverde, 2013; Harper et al. 2014).

En Panamá, se le ha prestado mayor atención a la pesca industrial, con relación a la artesanal, lo que ha llevado a tener mejores datos de esta actividad, con información recopilada desde los años 1950, ya que esta se desembarca en un número limitado de puertos, sobre todo en Vacamonte (Valverde, 2013; Harper et al. 2014; Castrellón \& Bucarán, 2020).

Por su parte la pesca artesanal, que descarga sus productos en unos 240 sitios, entre puertos y desembarcaderos (ARAP, 2017), se ha convertido en un problema para recopilación de datos, sobre todo por las debilidades institucionales de falta de recurso y personal capacitado (Castrellón \& Bucarán, 2020).

Probablemente, la mayor cantidad de información biológica y de pesca artesanal de escama está circunscrita a áreas protegidas, como el golfo de Montijo y el Parque Nacional Coiba. Es así como se cuenta con información sobre esciénidos, lutjánidos y escómbridos (Vega et al. 2008; Bonilla-Gómez et al. 2013; Vega et al. 2013), pargo, cherna y dorado (Guzmán et al. 2015; Vega et al. 2016) y tiburón
(Robles et al. 2015; Guzmán et al. 2020).

Esta información, más allá de su incorporación para el desarrollo de planes de manejo de áreas protegidas, no ha incidido en la toma de decisiones sobre la gestión pesquera en el país. Por ejemplo, se carece de legislaciones sobre tallas mínima de captura en peces, cuotas y protección de áreas de crianza.

El golfo de Parita, a pesar de que cuenta con áreas importantes de pesca, carece de información que apoye a los tomadores de decisiones para crear políticas de manejo para mejorar la administración pesquera en la zona. En este sentido, se analiza la composición de los desembarques de la actividad artesanal y la relación entre la estructura de tallas y la longitud media reproductiva de las especies más abundantes.

\section{MATERIAL Y MÉTODOS}

Los muestreos se realizaron en los puertos de Boca de Parita $\left(8^{\circ} 01^{\prime}\right.$ 06.13 " N y $80^{\circ} 27$ ' $15.38^{\prime \prime}$ W) y El Agallito (7० 59'27.63" N y 80²4'01.04" $\mathrm{W})$, donde se desembarcan capturas procedentes de al menos 30 puntos de pesca donde tienen actividad las embarcaciones artesanales (Fig. 1). 


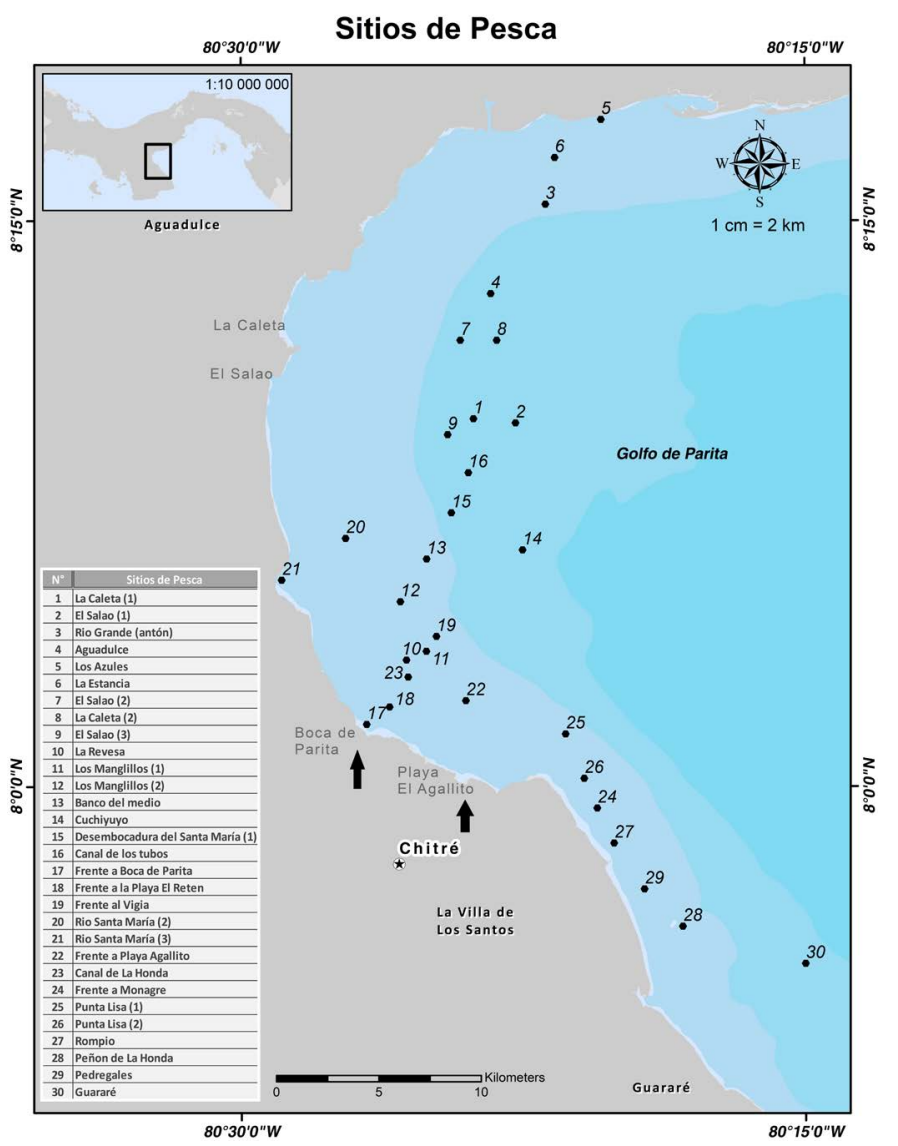

Fig. 1. Sitios de pesca artesanal en la Bahía de Parita. Las flechas indican los puntos de desembarque: Boca de Parita y el Agallito

Fig. 1. Artisanal fishing sites in Parita Bay. Arrows indicate landing points: Boca de Parita and Agallito durante tres días a la semana, cada semana.

En cada visita se tomaron muestras de las canastas. Cuando había pocos especímenes se registraron todos $\mathrm{y}$ cuando el desembarque incluía muchos individuos de algunos grupos se tomaban ejemplares al azar de diferentes cestas. Cada ejemplar fue identificado utilizando literatura especializada (Bussing \& López, 1993; Fischer et al. 1995; Robertson \& Allen, 2015) y se le registró el largo total con un ictiómetro $(\mathrm{cm})$ y peso total con una balanza digital $(0.1 \mathrm{~g})$.

Las empresas comercializadoras, en ambos puertos, suministraron el precio de pago al pescador de los productos pesqueros, lo que

Muestreos: los datos de desembarque 2011-2015 fueron proporcionados por la Autoridad de los Recursos Acuáticos de Panamá (ARAP), sede de Herrera, los cuales incluían descargues (kg), especies, artes, fechas y sitios de pesca. Para tallas y pesos se realizaron muestreos entre junio y diciembre de 2015 , con visitas diarias a cada puerto permitió identificar las especies que pertenecer a primera, segunda y tercera categoría. Para comparar biomasa desembarcada por año, en ambos puertos, se aplicó la prueba Kruskal Wallis para un $\alpha=0.05$ (Zar, 2009). 


\section{RESULTADOS}

Entre 2011 y 2015 se desembarcaron en los puertos de Boca de Parita y El Agallito 2760 toneladas en pesca de escama. Del total, $2060(74.5 \%)$ correspondió al primer puerto y $700(25.5 \%)$ al segundo. Por año de desembarque se aprecia un ligero aumento en el 2013, comparado con los restantes años en ambos puertos, aunque sin diferencias significativas $(P>0.05)$ (Fig. 2).
Composición de los desembarques: en total se analizaron 5342 ejemplares incluidos en 38 especies (fig. 3, cuadro 1). Con base en esa cantidad, los mayores aportes lo hicieron las familias Sciaenidae, Lutjanidae, Scombridae y Carangidae y por especies, las que presentaron mayor cantidad de individuos fueron Cynoscion phoxocephalus (Sciaenidae), Scomberomorus sierra (Scombridae) y Lutjanus guttatus (Lujanidae) (Fig. 3).

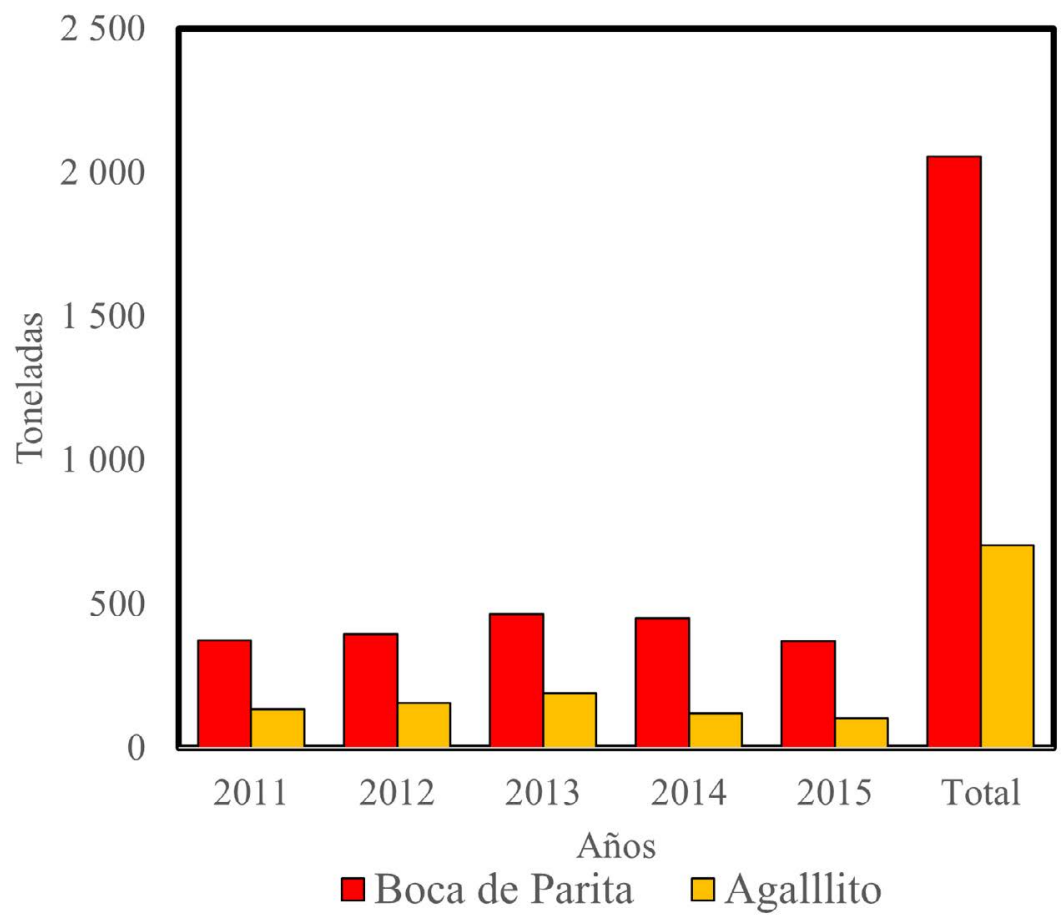

Fig. 2. Biomasa en toneladas registrada por puerto de desembarque. Capturas procedentes del golfo de Parita, Pacífico de Panamá. Fuente: empresas comercializadoras

Fig. 2. Biomass recorded by landing port: Catches from Parita Gulf in the Panamanian Pacific. Source: trading companies 


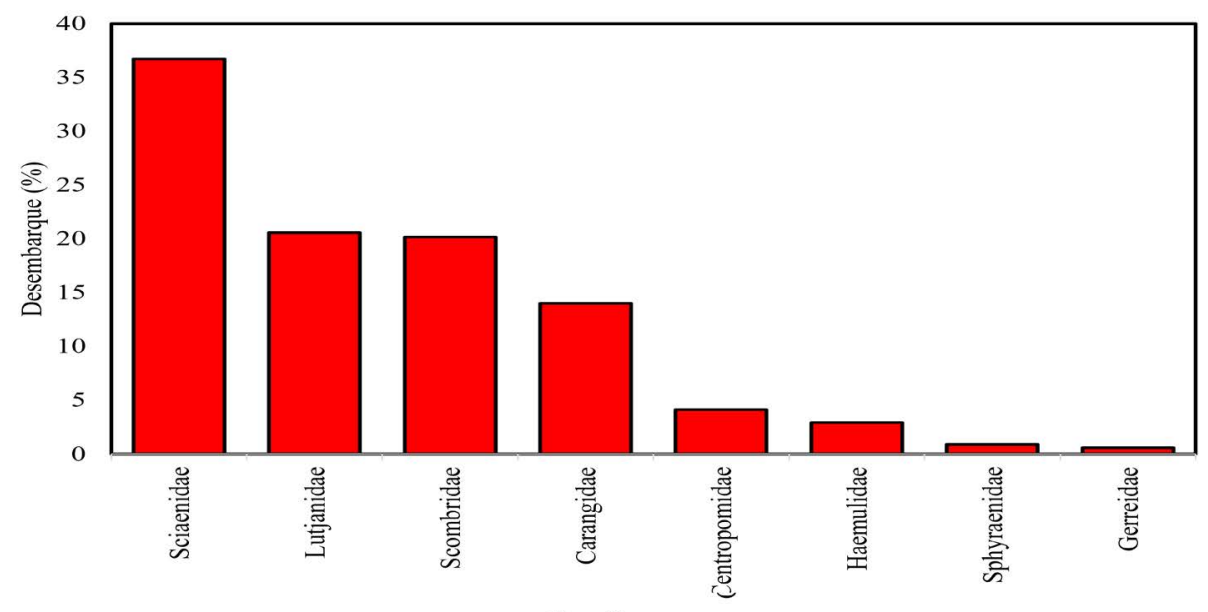

Familias

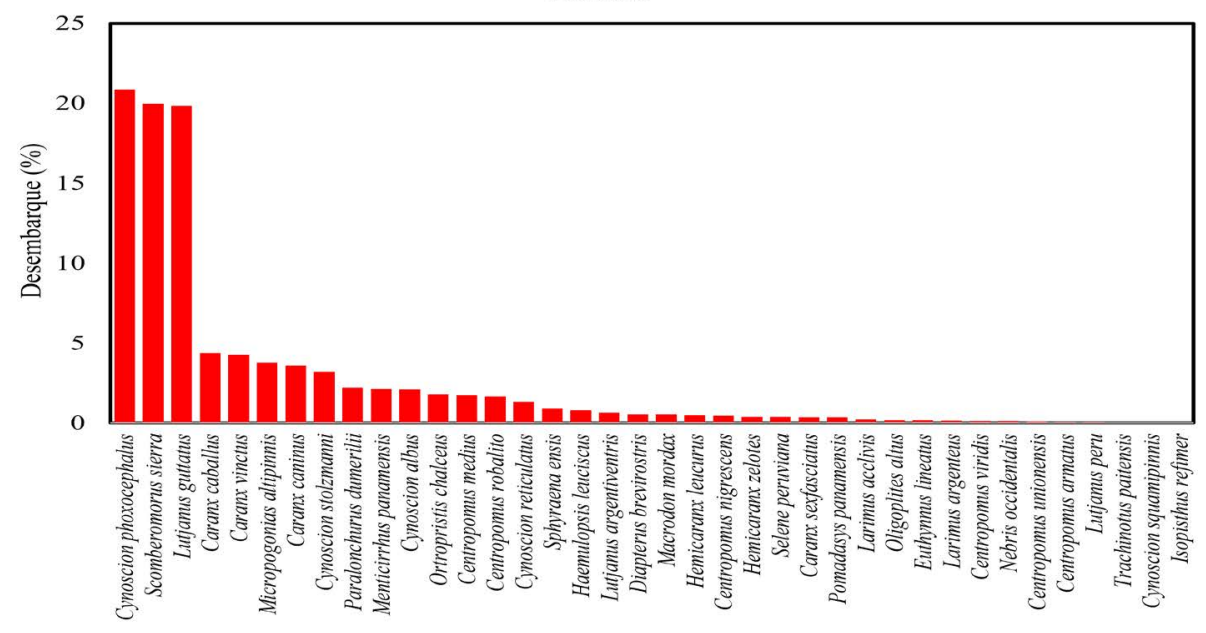

Especies

Fig. 3. Composición de los desembarques por familia y especies. Muestreos realizados en empresas comercializadoras de Boca de Parita y El Agallito, Pacífico de Panamá Fig. 3. Catch composition by family and species. Sampling was carried out at trading companies from the ports of Boca de Parita and Agallito, in the Panamanian Pacific

Tallas y pesos: el $78 \%$ de las especies desembarcadas se incluyen en las familias Sciaenidae (13), Carangidae (9) y Centropomidae (6). El $74 \%$ se ubicó en longitudes y pesos inferiores a $10 \mathrm{c} 40 \mathrm{~cm}$ y $500 \mathrm{~g}$, excepto aquellas que alcanzan tamaños relativamente grandes, que sobrepasan los $90 \mathrm{~cm}$ de longitud total, como algunas corvinas (Cynoscion albus), róbalos (Centropomus nigrescens y C. viridis), pargo (Lutjanus peru) y organismos pelágicos como Scomberomorus sierra y Euthynnus lineatus (Cuadro 1). 
Cuadro 1. Tallas y pesos de las principales especies desembarcadas en los puertos de Boca de Parita y El Agallito, Pacífico de Panamá

Table 1. Size and weight of the main species landed in the ports of Boca de Parita and El Agallito, in the Panamanian Pacific

\begin{tabular}{|c|c|c|c|c|c|c|}
\hline \multirow[t]{2}{*}{ Familia } & \multirow[t]{2}{*}{ +Especie } & \multicolumn{2}{|c|}{ LT (cm) } & \multicolumn{2}{|c|}{ PT $(g)$} & \multirow[b]{2}{*}{$\mathbf{N}$} \\
\hline & & Media & DE & Media & DE & \\
\hline \multirow[t]{9}{*}{ Carangidae } & Caranx caballus & 29.79 & 4.41 & 328.65 & 195.01 & 234 \\
\hline & Caranx caninus & 26.38 & 3.99 & 262.06 & 129.51 & 192 \\
\hline & Caranx sexfasciatus & 27.53 & 7.85 & 334.63 & 491.66 & 19 \\
\hline & Caranx vinctus & 31.37 & 8.68 & 333.47 & 171.30 & 227 \\
\hline & Hemicaranx leucurus & 28.31 & 5.31 & 254.65 & 147.53 & 26 \\
\hline & Hemicaranx zelotes & 32.74 & 1.04 & 372.00 & 29.89 & 20 \\
\hline & Oligoplites altus & 45.40 & 2.63 & 849.50 & 124.43 & 10 \\
\hline & Selene peruviana & 31.10 & 2.93 & 318.50 & 76.16 & 20 \\
\hline & Trachinotus paitensis & 20.60 & & 114.00 & & 1 \\
\hline \multirow[t]{6}{*}{ Centropomidae } & Centropomus armatus & 34.88 & 2.46 & 368.54 & 56.70 & 4 \\
\hline & Centropomus medius & 46.10 & 7.75 & 825.51 & 420.68 & 104 \\
\hline & Centropomus nigrescens & 40.05 & 5.93 & 671.37 & 267.02 & 24 \\
\hline & Centropomus robalito & 30.26 & 3.30 & 351.57 & 168.00 & 89 \\
\hline & Centropomus unionensis & 32.30 & 3.40 & 476.27 & 147.85 & 5 \\
\hline & Centropomus viridis & 63.50 & 10.45 & 1731.15 & 594.41 & 6 \\
\hline Gerreidae & Diapterus brevirostris & 25.03 & 1.74 & 309.95 & 66.14 & 30 \\
\hline \multirow[t]{3}{*}{ Haemulidae } & Haemulopsis leuciscus & 24.07 & 3.77 & 242.99 & 157.55 & 42 \\
\hline & Orthopristis chalceus & 22.63 & 1.36 & 197.45 & 45.95 & 96 \\
\hline & Pomadasys panamensis & 22.21 & 3.33 & 668.45 & 192.35 & 19 \\
\hline \multirow[t]{3}{*}{ Lutjanidae } & Lutjanus argentiventris & 28.04 & 3.42 & 372.38 & 149.87 & 35 \\
\hline & Lutjanus guttatus & 29.86 & 4.80 & 366.39 & 180.67 & 1060 \\
\hline & Lutjanus peru & 43.67 & 2.36 & 1200.06 & 256.11 & 3 \\
\hline \multirow[t]{13}{*}{ Sciaenidae } & Cynoscion albus & 42.45 & 19.04 & 1284.05 & 2631.15 & 113 \\
\hline & Cynoscion phoxocephalus & 34.08 & 3.92 & 486.51 & 154.01 & 1114 \\
\hline & Cynoscion reticulatus & 33.51 & 3.89 & 408.41 & 155.95 & 71 \\
\hline & Cynoscion squamipinnis & 42.00 & & 453.59 & & 1 \\
\hline & Cynoscion stolzmanni & 37.74 & 4.46 & 510.87 & 183.80 & 171 \\
\hline & Isopisthus remifer & 29.00 & & 215.00 & & 1 \\
\hline & Larimus acclivis & 24.67 & 1.97 & 263.86 & 102.46 & 13 \\
\hline & Larimus argenteus & 28.21 & 1.87 & 307.79 & 107.86 & 7 \\
\hline & Macrodon mordax & 33.19 & 5.24 & 345.53 & 182.27 & 30 \\
\hline & Menticirrhus panamensis & 32.69 & 4.01 & 480.99 & 204.98 & 114 \\
\hline & Micropogonias altipinnis & 30.56 & 3.51 & 346.54 & 152.61 & 202 \\
\hline & Nebris occidentalis & 29.70 & 2.87 & 277.03 & 81.57 & 6 \\
\hline & Paralonchurus dumerilii & 27.51 & 2.88 & 256.55 & 205.24 & 118 \\
\hline \multirow[t]{2}{*}{ Scombridae } & Euthynnus lineatus & 53.70 & 2.06 & 1944.80 & 336.50 & 10 \\
\hline & Scomberomorus sierra & 48.51 & 6.90 & 596.98 & 298.16 & 1068 \\
\hline Sphyraenidae & Sphyraena ensis & 57.11 & 7.20 & 797.96 & 210.32 & 48 \\
\hline
\end{tabular}


La distribución de tallas, en las los desembarques estuvo compuesto especies con un tamaño de muestra superior a 100 ejemplares y para las cuales se encontró información sobre la longitud media reproductiva $\left(\mathrm{L}_{50}\right)$, refleja que solo en Caranx vinctus y Centropomus medius, más del $80 \%$ de

por individuos maduros, en Lutjanus guttatus y Scomberomorus sierra el $49 \%$ y $53 \%$, y en Cynoscion phoxocephalus, Caranx caballus, y Cynoscion albus, el porcentaje fue de 19, 5 y 0 , respectivamente (Fig. 4).
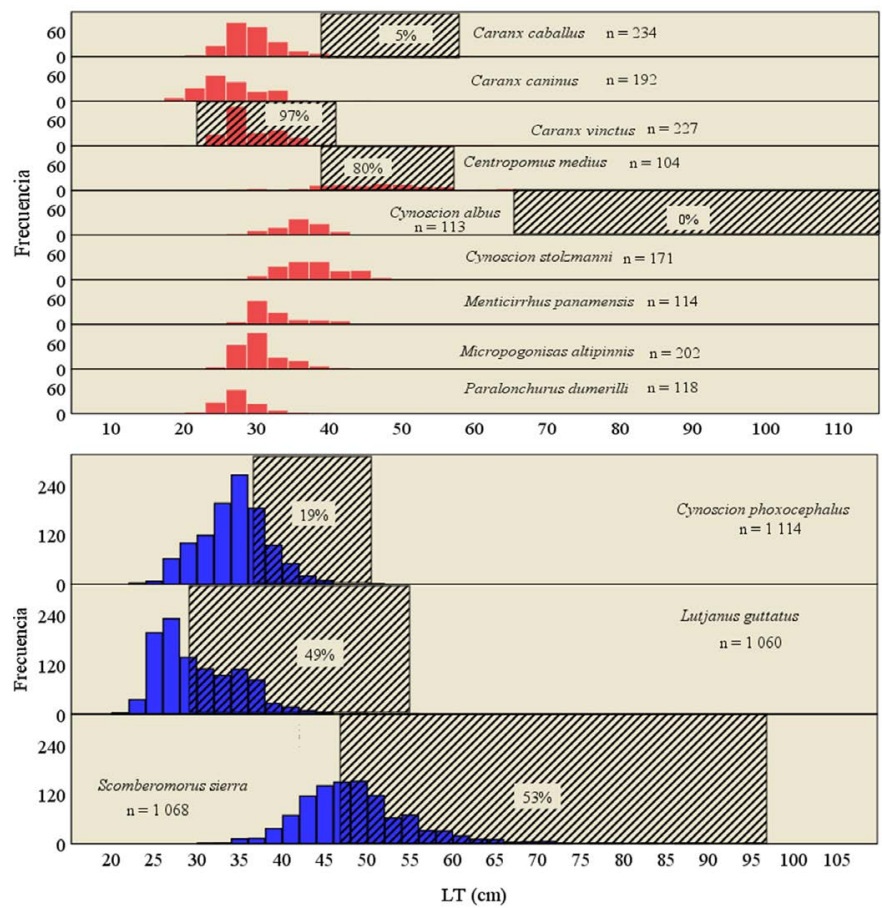

Fig. 4. Estructura de tallas de las especies con más de 100 individuos en los muestreos de desembarque. Muestreos realizados en los puertos de Boca de Parita y El Agallito, Pacífico de Panamá, entre junio y diciembre de 2015. El sombreado representa el porcentaje de individuos ubicados por encima de la talla media reproductiva $\left(\mathrm{L}_{50}\right)$. La $\mathrm{L}_{50}$, para algunas especies, se obtuvo de Aguirre-Villaseñor et al. 2006, Vega et al. 2011, Robles \& Montes, 2011, Espino-Barr et al. 2012, Mair et al. 2012. Fig. 4. Size structure of species with landing site samples of over 100 individuals. Sampling conducted at the ports of Boca de Parita and Agallito, in the Panamanian Pacific, between June and December of 2015. Shading represents the percentage individuals over mean reproductive size (L50). The (L50) parameter was obtained from Aguirre-Villaseñor et al. 2006, Vega et al. 2011, Robles \& Montes, 2011, Espino-Barr et al. 2012, Mair et al. 2012 
Comercialización: la venta de los productos pesqueros se realiza según categorías, donde el pescado de primera incluye a los pargos del género Lutjanus (Lutjanidae), corvinas delgrupoCynoscion (Sciaenidae) y róbalos (Centropomidae); el de segunda incluye a Scomberomorus sierra, la cojinúa (Caranx caballus), el jurel (Caranx caninus) y algunos esciénidos (Menticirrhus panamensis y Micropogonia altipinnis).

El resto de los desembarques analizados se agrupan como producto de tercera clase o revoltura. Los pagos a pescadores, en dólares estadounidenses, según grupo de comercialización estuvo entre 1.50 y 1.25 , para pescado de primera, el de segunda entre $0.80 \mathrm{y}$ 0.60 y el de tercera de 0.15 y 0.60 , según época del año, donde los mejores precios se obtienen hacia las festividades de Semana Santa.

Según estas categorías, al analizar la contribución en peso de los desembarques el 54\% correspondió a producto de primera clase, el $41 \%$ a segunda y el restante $5 \%$ a tercera o revoltura. En número de individuos la contribución por grupo de comercialización fue de $52 \%$, $40 \%$ y $8 \%$, respectivamente (Fig. 5).

\section{DISCUSIÓN}

Para el golfo de Montijo, registros oficiales (2008-2014), indican que los mayores desembarques de productos pesqueros, aunque en menor magnitud,

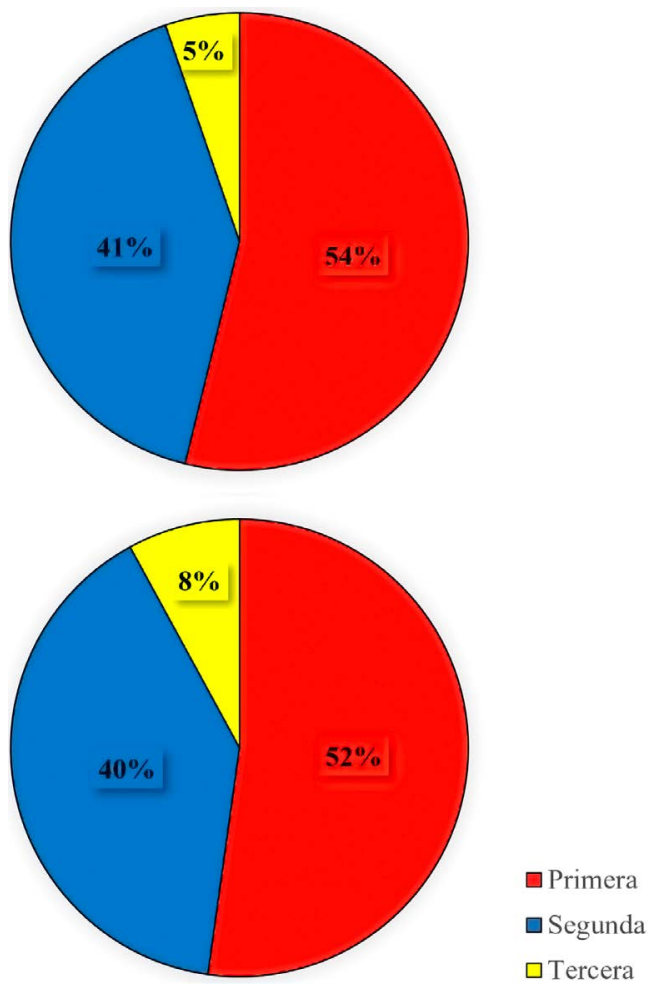

Fig. 5. Aporte porcentual de las especies, según categoría de comercialización. A: distribución según peso de los individuos, B: distribución según cantidad de individuos. Muestreos realizados en empresas comercializadoras de Boca de Parita y El Agallito, Pacífico de Panamá

Fig. 5. Species percentage contribution according to marketing category. A: weight distribution of individuals, B: landed amount distribution. Sampling was carried out at marketing companies from the ports of Boca de Parita and Agallito, in the Panamanian Pacific 
se presentaron en el año 2013, con 392 toneladas (Virola, 2014), lo cual coincide con la tendencia observada en Boca de Parita y el Agallito.

Es importante resaltar que la información del golfo de Montijo es oficial, por lo que pueden tener un sesgo asociado a los subregistros por la poca sistematicidad en la captura de la información, a diferencia de los datos obtenidos de las empresas en Boca de Parita y el Agallito, donde el reporte es más confiable, ya que capturan toda la información de desembarque, debido a que están vinculados a procesos contables de compra y venta.

El total de especies registradas es bajo con relación a otros estudios desarrollados en ambientes similares; para el área costera entre San Lorenzo y Pixvae, en el golfo de Chiriquí, se comunicaron 111 especies asociados a la pesca Ribereña (Vega et al. 2011), para Manglares de David 142 (Robles \& Montes 2011) y para el golfo de Montijo 188 (Vega, 2014).

Lo anterior se explica en función del mayor esfuerzo de muestreo realizado en estos sistemas, en comparación con el presente trabajo, sobre todo porque no se completó un ciclo anual, lo que puede incidir en la ausencia de especies que se incorporan a la pesca en la temporada seca (enero a abril), y el inicio de las lluvias (abril a junio).

Tanto para Boca de Parita como para el Agallito, las familias con mayor representatividad numérica en los desembarques fueron Sciaenidae, Scombridae y Lutjanidae, en las cuales algunas de sus especies se capturan en áreas cercanas a la costa y a las zonas estuarinas.

Es así como, las especies con mayor cantidad de individuos en las capturas fueron Cynoscion phoxocepahalus, Scomberomorus sierra y Lutjanus guttatus, las cuales son típicas de la pesca que ocurren en la parte externa de los estuarios (Vega et al. 2008; Robles \& Montes, 2011; Vega et al. 2013; 2014) o a lo largo de frentes de playas entre San Lorenzo y Pixvae, en el golfo de Chiriquí (Vega et al. 2011), excepto Lutjanus guttatus que se pesca desde áreas estuarinas hasta montículos submarinos, islas e islotes en la plataforma continental (Robles \& Montes 2011; Vega et al. 2016).

La longitud de captura está directamente relacionada a la que alcanza el pez, la zona y profundidad de la pesca y el arte utilizado, que define una selectividad (Christiansen et al. 2020). De las 13 especies a las cuales se le analizó la estructura de tallas, solo para siete se encontró información publicada sobre media reproductiva $\left(\mathrm{L}_{50}\right)$.

El análisis de las tallas de captura en función de la $\mathrm{L}_{50}$ refleja que solo en tres de las 13 especies analizadas, más del $50 \%$ de los individuos superaron la media reproductiva, en las restantes nueve, el porcentaje fue inferior a $50 \%$, donde Cynoscion albus y Caranx caballus presentaron en más del $95 \%$ de los 
ejemplares desembarcados longitudes por debajo de la $\mathrm{L}_{50}$.

En el caso de C. caballus, existe reporte para isla de las Perlas, golfo de Panamá, donde se cataloga a esta especie como sobreexplotada y se recomienda elevar la talla mínima de captura a valores que aumenten el potencial reproductivo de la población, para garantizar la sostenibilidad del recurso (Mair et al. 2012).

La pesquería que se desarrolla en el golfo de Parita es multiespecífica, donde la clasificación por categorías de comercialización refleja que más del $50 \%$ de los desembarques, en cantidad de individuos y en peso, estuvo conformado por especies de primera (pargos, corvinas y robalos) y casi $40 \%$ de segunda, principalmente Scomberomorus sierra.

Esto crea la necesidad de establecer una gestión pesquera que incorpore, no solo regulaciones sobre tallas de captura, sino la protección de sitios para especies que presentan distribución diferencial de longitudes y edades, con migraciones ontogénicas o de desove, como es el caso de los pargos (Grüss et al. 2019; Roos et al. 2020).

Según Froese (2004), tres indicadores sencillos para contrarrestar la sobre pesca implica tener como objetivo: el $100 \%$ con talla óptima de captura y de peces maduros y $0 \%$ de megareproductores, aunque sin normativa sobre talla máxima, es razonable la captura de un $30 \%$ a $40 \%$ de estos.
Sin duda es poca la información para analizar el cumplimiento de estos indicadores, ya que no existe en Panamá, programas de monitoreos, ni de ordenamiento pesquero integrales, que permita lograr un manejo de la pesca artesanal. Aun así, la información generada en este estudio permite, por lo menos en algunas especies de interés pesquero en el golfo de Parita, dar luces sobre el comportamiento de las capturas.

Esto nos lleva a concluir que sin duda es necesario buscar alternativas para mejorar el comportamiento de las capturas, a través de normativas que apunten a evitar la sobrepesca y sobreexplotación de los recursos pesqueros, como pueden ser regulaciones sobre tallas mínimas y máximas, sobre zonas $\mathrm{y}$ artes de pesca permitidos.

Es importante resaltar la necesidad de ampliar el muestreo a los meses no incluidos en este estudio y desarrollar campañas a bordo de embarcaciones con el objetivo de contrastar las capturas versus los desembarques, además de establecer la talla mínima reproductiva en las especies para las cuales no se conoce este parámetro.

\section{CONCLUSIONES}

Los desembarques de la pesca artesanal proveniente del golfo de Parita son multiespecíficos, donde, por lo menos en la segunda mitad del año, dominaron en las capturas las especies 


\section{Cynoscion phoxocephalus (Sciaeni-} dae), Scomberomorus sierra (Scombridae) y Lutjanus guttatus (Lutjanidae), catalogadas como de primera y segunda categoría, según el precio de venta.

Similar a lo que ocurre en otras localidades del Pacífico panameño, un porcentaje importante de las capturas estuvo representadas por individuos que no se han reproducido, lo que puede llevar a la pesquería a una sobreexplotación, por lo que es necesario establecer medidas de manejo que garanticen la sostenibilidad.

\section{AGRADECIMIENTOS}

Se agradece a los pescadores y a las empresas comercializadoras de productos pesqueros que operan desde los puertos de Boca de Parita y El Agallito, por permitir el manejo de sus capturas y por facilitar los datos de desembarque, y a los revisores anónimos que contribuyeron a mejorar el presente artículo.

\section{REFERENCIAS}

Aguirre-Villaseñor, H., Morales-Bojórquez, E., Morán-Angulo, R. E., Madrid-Vera, J. \& Valdez-Pineda, M. C. (2006). Biological indicators for the Pacific sierra (Scomberomorus sierra) Fishery in the southern Gulf of California, México. Ciencias Marinas, 32(3),471-484. https://doi.org/10.7773/cm.v32i3.1130

Autoridad Nacional del Ambiente (2009). Plan de manejo del Parque Nacional
Coiba. Panamá, Smithsonian Tropical Research Institute.

ARAP. Autoridad de los Recursos Acuáticos de Panamá (2017). Plan nacional de acción para la pesca sostenible en $\mathrm{Pa}$ namá. Panamá. Autoridad de Recursos Acuáticos de Panamá.

Arreguín-Sánchez, F. \& Arcos-Huitrón, E. (2011). La pesca en México: estado de la explotación y uso de los ecosistemas. Hidrobiológica, 21(3), 431-462.

Bonilla-Gómez, J. L., Robles, Y. A. \& Vega, A. J. (2013). Length-weight relationship and biological information of the yellow snapper Lutjanus argentiventris from a tropical estuary: Río Caté, Gulf of Montijo, Panama. J. Appl. Ichthyol., 34, 227 229. https://doi.org/10.1111/jai.12258

Bussing, W. A. \& López, M. I. (1993). Peces demersales y pelágicos costeros del Pacífico de Centroamérica meridional. Costa Rica: Universidad de Costa Rica.

Castrellón, M. \& Bucarán, S. J. (2020). Diagnóstico integral del sector pesca $\mathrm{y}$ acuicultura de la República de Panamá. Panamá. BID, Nota Técnica No IDB-TN- 02011.

Christiansen, H. M., Switzer, T. S., Keenan, S. F., Tyler-Jedlund, A. J. \& Winner, B. L. (2020). Assessing the Relative Selectivity of Multiple Sampling Gears for Managed Reef Fishes in the Eastern Gulf of Mexico. Mar. Coastal Fish.: Dynamics, Manage., Ecosyst. Sci., 12, 322-338. https://doi.org/10.1002/mcf2.10129

Espino-Barr, E., Nava-Ortega., R. A., Gallardo-Cabello, M., Cabral-Solís, E. G., Puente- Gómez, M. \& García-Boa, A. (2012). Aspects of Scomberomorus sierra fishery from the Coast of Colima, México. Ciencia Pesquera, 20(1), 77-88.

Fischer, W., Krupp, F., Schneider, W., Sommer, C., Carpenter, K. E. \& Niem, V. H. (1995). Guía FAO para la identificación de especies para los fines de la 
pesca. Pacífico centro-oriental, Vol. II y III. Italia: FAO.

Froese, R. (2004). Keep it simple: Three indicators to deal with overfishing. Fish. Fish., 5, 86-91. https://doi.or$\mathrm{g} / 10.1111 / \mathrm{j} .1467-2979.2004 .00144 . \mathrm{x}$

González-Becerril, A., Espino-Barr, E., Cruz-Romero, M. \& Ruíz-Luna, A. (2000). Determinación de la unidad de esfuerzo de pesca en una pesquería artesanal ribereña en Manzanillo, Colima, México. Ciencias Marinas, 26(1), 113-124. https://doi. org/10.7773/cm.v26i1.568

Grüss, A., Biggs, C. R., Heyman, W. D. \& Erisman, B. (2019). Protecting juveniles, spawners or both: a practical statistical modelling approach for the design of marine protected areas. J. Appl. Ecol., 56, 2328-2339. https://doi. org/10.1111/1365-2664.13468

Gutiérrez-Zavala, R. M. \& Cabrera-Mancilla, E. (2012). La pesca ribereña de Guerrero. México. Instituto Nacional de Pesca.

Guzmán, H. M., Cipriani, R., Vega, A. J. \& Morales-Saldaña, J. M. (2020). Fisheries and conservation assessment of sharks in Pacific Panama. Aqua. Cons. Mar. Fresh. Ecos., 30(2), 315-330. https://doi.org/10.1002/aqc.3245

Guzmán, H. M., Diaz-Ferguson, E., Vega, A. J. \& Robles, Y. A. (2015). Assessment of the dolphinfish Coryphaena hippurus (Perciformes: Coryphaenidae) fishery in Pacific Panama. Rev. Biol. Trop., 63(3), 705-716. https://doi. org/10.15517/rbt.v63i3.15487

Guzmán-Mora, A. G. \& Molina-Ureña, H. (2008). La pesquería artesanal de peces en golfo Dulce, Costa Rica. Proc. 60th Gulf Caribb. Fish. Inst.,137-142.

Harper, S., Guzmán, H. M., Zylich, K. \& Zeller, D. (2014). Reconstructing Panama's Total Fisheries Catches from 1950 to 2010: Highlighting Data Deficiencies and Management Needs. Mar.
Fish. Rev., 72(1-2), 51-65. https://doi. org/10.7755/MFR.76.1_2.3

Liu, O. R., Thomas, L. R., Clemence, M., Fujita, R., Kritzer, J. P., McDonald, G. \& Szuwalski, C. (2016). An evaluation of harvest control methods for fishery management. Rev. Fish. Sci. Aquacult., 24(3),244-263. https://doi.org/10.1080 /23308249.2016.1161002

Mair, J. M., Cipriani, R., Guzman, H. M. \& Usan, D. (2012). Fishery of the Green Jack Caranx caballus (Osteichytes: Carangidae) in Las Perlas Archipelago, Pacific Panama. Rev. Biol. Trop., 60(3), 1271-1288. https://doi.org/10.15517/rbt.v60i3.1806

Maté, J. (2005). Análisis de la situación de la pesca en los golfos de Chiriquí y de Montijo. Panamá. The Nature Conservancy.

Robertson, D. R. \& Allen, G. R. (2015). Peces costeros del Pacífico Oriental Tropical: sistema de información en línea. Versión 2.0. Instituto Smithsonian de Investigaciones Tropicales, Panamá. http://biogeodb.stri.si.edu/sftep/

Robles, Y. A., Montes, L. A. \& Vega, A. J. (2015). Caracterización de la captura de tiburones por la pesca artesanal en los manglares de David, golfo de Chiriquí, Pacífico de Panamá. Tecnociencia, 17(1), 11-30.

Robles, Y. A. \& Montes, L. (2011). Caracterización biológica de la pesquería artesanal que se desarrolla en el golfo de Chiriquí. Panamá, Universidad de Panamá.

Roos, N. C., Taylor, B. M., Carvalho, A. R. \& Longo, G. O. (2020). Demography of the largest and most endangered Brazilian parrotfish, Scarus trispinosus, reveals overfishing. End. Sp. Res., 41, 319-327. https://doi.org/10.3354/esr01024

Valverde, A. (2013). Producción y comercialización de la pesca artesanal o de pequeña escala en Panamá desde 1995 al 2008. Centros, 2(1),115-131. 
Vázquez-Hurtado, M., Maldonado-García, M., Lechuga-Devéze, C. H., Acosta-Salmón, H. \& Ortega-Rubio, A. (2010). La pesquería artesanal en la Bahía de La Paz y su área oceánica adyacente (golfo de California, México). Ciencias Marinas, 36(4), 433-444.

Vega, A. J. (2014). Plan de aprovechamiento pesquero sostenible del área de recursos manejados humedal golfo de Montijo (ARMHGM). Panamá. ANAM-CI-MARVIVA-NATURA.

Vega, A. J., Robles, Y. A. \& Maté, J. L. (2016). La pesca artesanal en el Parque Nacional Coiba y Zonas de influencia. Panamá, SENACYT, Fundación MARVIVA.

Vega, A. J., Quezada, F. \& Robles, Y. (2013). Aspectos biológicos y pesqueros de Scomberomorus sierra (Perciformes: Scombridae) en el golfo de Montijo, Pacífico de Panamá, Tecnociencia, 15(2), 53-70.
Vega, A. J., Robles, Y. A. \& Cipriani, R. (2011). Estudios biológicos-pesqueros en el golfo de Chiriquí; Pacífico de Panamá. Panamá. SENACYT, Fundación MARVIVA, Conservación Internacional.

Vega, A. J., Robles, Y. A., Boniche, S. \& Rodríguez, M. (2008). Aspectos biológico-pesqueros del género Cynoscion (Pisces: Sciaenidae) en el golfo de Montijo, Pacífico Panameño. Tecnociencia, 10(2), 9-26.

Virola, B. (2014). Análisis histórico de los desembarques pesqueros en el golfo de Montijo, Pacífico panameño: Periodo 2008-2013. (Tesis de licenciatura no publicada), Universidad de Panamá, Panamá.

Zar, J. H. (2009). Biostatistical Analysis. EE. UU.: Prentice Hall. 